CERN-PH-TH/2006-259

IFIC $/ 06-45$

\title{
Spontaneous chiral symmetry breaking in QCD: a finite-size scaling study on the lattice
}

\author{
Leonardo Giusti ${ }^{a}$ and Silvia Necco ${ }^{b}$ \\ ${ }^{a}$ CERN, Physics Department, TH Division, CH-1211 Geneva 23, Switzerland \\ ${ }^{b}$ Instituto de Física Corpuscular, CSIC-Universitat de València \\ Apartado de Correos 22085, E-46071 Valencia, Spain
}

\begin{abstract}
Spontaneous chiral symmetry breaking in QCD with massless quarks at infinite volume can be seen in a finite box by studying, for instance, the dependence of the chiral condensate from the volume and the quark mass. We perform a feasibility study of this program by computing the quark condensate on the lattice in the quenched approximation of QCD at small quark masses. We carry out simulations in various topological sectors of the theory at several volumes, quark masses and lattice spacings by employing fermions with an exact chiral symmetry, and we focus on observables which are infrared stable and free from mass-dependent ultraviolet divergences. The numerical calculation is carried out with an exact variance-reduction technique, which is designed to be particularly efficient when spontaneous symmetry breaking is at work in generating a few very small low-lying eigenvalues of the Dirac operator. The finite-size scaling behaviour of the condensate in the topological sectors considered agrees, within our statistical accuracy, with the expectations of the chiral effective theory. Close to the chiral limit we observe a detailed agreement with the first Leutwyler-Smilga sum rule. By comparing the mass, the volume and the topology dependence of our results with the predictions of the chiral effective theory, we extract the corresponding low-energy constant.
\end{abstract}




\section{Introduction}

Spontaneous symmetry breaking plays a central rôle in our understanding of the strong interactions. In QCD with (a small number of) $N_{f}$ light flavors the standard expectation is that the $S U\left(N_{f}\right)_{L} \times S U\left(N_{f}\right)_{R}$ chiral symmetry group breaks spontaneously to $S U\left(N_{f}\right)_{L+R}$ with the formation of a non-zero quark condensate. There are many evidences that support this picture [1-14], but a conclusive study with a reliable determination of the condensate is still missing 1 .

The lattice formulation of gauge theories is at present the only approach where QCD can be defined non-perturbatively. Computations in this regime are performed by numerical simulations, and therefore limited to be at finite volume and lattice spacing. The spontaneous formation of a quark condensate $\Sigma$ at infinite volume can be detected in a finite box by studying the properties of the long-range correlations left in the system, a technique widely used in statistical mechanics [16]. In QCD the simplest observable to consider is maybe the chiral condensate generated by adding a small quark mass $m$ to the action. This explicit breaking of chiral symmetry gives also rise to additive ultraviolet divergences which make the definition of the condensate ambiguous. The crucial observation here is that the ultraviolet divergences are independent on the volume $V$ and, in a regularization that preserve chiral symmetry, their magnitude is suppressed by a factor $1 / V$ with respect to the contributions from the long-range correlations [17]. At any given lattice spacing the value of $\Sigma$ can then be extracted with arbitrary precision from a finite-size scaling study of the condensate if the volumes are large enough.

In a finite box it is also interesting to consider QCD in a fixed topological sector, i.e. with the functional integral restricted to gauge-field configurations with a given topological charge. Recent progress in the understanding of topology on the lattice [1820] allows to show that the chiral condensate has the same ultraviolet divergences in the full theory and at fixed topology. It can be made ultraviolet finite with the very same renormalization constants and subtraction coefficients. On the other hand the chiral effective theory makes definite predictions, with no extra free parameters, for the topology dependence of the infrared contribution to condensate [17,21,22], predictions which can be directly tested against lattice QCD results.

Despite of many efforts dedicated to implement this program in the lattice community $[7,10,11]$, a reliable determination of the QCD chiral condensate is still missing. The main reasons being the limitations in our ability of going beyond the quenched approximation in the numerical simulations of QCD, and the large numerical instabilities in the commonly used estimators of the chiral condensate at finite volume [11]. The first problem is being solved over the last few years thanks to the development of new algorithms for simulating dynamical fermions [23,24]. The implementation of some of these new ideas to simulate dynamical Ginsparg-Wilson fermions is progressing

\footnotetext{
${ }^{1}$ For a recent review of the experimental implications of spontaneous symmetry breaking in QCD see Ref. [15] and references therein.
} 
quite fast. First results were already presented [25-28]. The second problem arises in computations of correlation functions at finite volume in the mass range $m \Sigma V \lesssim 1$. The long-range correlations left in the system generate small eigenvalues of the Dirac operator of order $1 / V[17]$. The latter give rise to extremely large fluctuations in the commonly used statistical estimators of correlation functions, and make it difficult to control the statistical errors $[11,29]$.

In this paper we address the second problem. We introduce numerical estimators of the condensate which are infrared stable and free from mass-dependent additive ultraviolet divergences. This progress builds on the recent understanding of the renormalization properties of the spectral density of the Dirac operator [30]. The form of these estimators represents and explicit example of how the informations from the chiral effective theory can be used not only for the interpretation/extrapolation of the data, but also to design more efficient numerical algorithms.

We apply these ideas to the computation of the chiral condensate in the quenched approximation of QCD with Neuberger fermions. For cubic lattices with linear extensions $L \gtrsim 1.5 \mathrm{fm}$, we observe a finite-size scaling behaviour of the condensate compatible with a theory which exhibits spontaneous symmetry breaking at asymptotically large volumes. The topology dependence of the data supports the predictions of the (quenched) effective theory. Close to the chiral limit we observe a detailed agreement with the first Leutwyler-Smilga sum rule [17]. Eventually we extract the value of the low-energy constant $\Sigma$ by matching the chiral effective theory formulas with the lattice results.

The paper is organized as follows. In Sec. 2 we discuss the ultraviolet properties of the chiral condensate with Neuberger fermions; in Sec. 3 we summarize the predictions of the effective theory for its volume, mass and topology dependence; in Sec. 4 we give details of the numerical computation, and in Sec. 5 we compare our results with the predictions of the effective theory. The appendices $\mathrm{A}$ and $\mathrm{B}$ are devoted to more technical details.

\section{The quark condensate with Ginsparg-Wilson fermions}

We consider QCD with $N_{f} \geq 2$ degenerate flavours regularized on an Euclidean lattice of spacing $a$ with the standard plaquette gauge action and with the NeubergerDirac operator [31]. The latter (see Appendix A for unexplained notations) satisfies the Ginsparg-Wilson relation [32]

$$
\gamma_{5} D+D \gamma_{5}=\bar{a} D \gamma_{5} D
$$

which guarantees an exact chiral symmetry

$$
\delta \psi=i \gamma_{5}(1-\bar{a} D) \psi, \quad \delta \bar{\psi}=\bar{\psi} \gamma_{5} i,
$$

of the massless fermion action at finite lattice spacing [33]. The Jacobian of the transformation is non-trivial, and the chiral anomaly is recovered à la Fujikwa [33-35], with 
the topological charge density operator defined as $[31,36,37]$

$$
a^{4} q(x)=-\frac{\bar{a}}{2} \operatorname{tr}\left[\gamma_{5} D(x, x)\right] .
$$

In a given gauge background the topological charge operator

$$
Q=a^{4} \sum_{x} q(x)
$$

is related to the index of the lattice Dirac operator $Q=n_{+}-n_{-}$where $n_{+}\left(n_{-}\right)$is the number of zero modes of $D$ with positive (negative) chirality. All the cumulants of the topological charge $Q$ are ultraviolet finite and thus the charge distribution [18-20]. Correlation functions of renormalized local operators inserted at a physical distance are therefore ultraviolet finite also in the theory restricted to a fixed topological sector. The action of the theory is invariant under the non-singlet chiral transformations analogous to the one in Eq. (2.2). They ensure that in the chiral limit the quark condensate renormalizes only multiplicatively

$$
\langle\bar{\psi} \psi\rangle=\lim _{a \rightarrow 0} Z_{S}\langle\bar{\psi} \tilde{\psi}\rangle
$$

where $\tilde{\psi}=\left\{\tilde{\psi}_{1}, \ldots, \tilde{\psi}_{N_{f}}\right\}, \bar{\psi}$ is defined analogously and the rotated field $\tilde{\psi}_{i}$ is given in the Appendix A. $Z_{S}$ is the logarithmic-divergent renormalization constant of the scalar density fixed with a given renormalization prescription. For our finite-size scaling study we are interested in breaking the chiral symmetry explicitly by adding a mass term to the action, which can be introduced by defining the massive Dirac operator as

$$
D_{m}=\left(1-\frac{\bar{a} m}{2}\right) D+m
$$

The quark condensate develops additional ultraviolet divergences which can be parameterized as

$$
-\frac{Z_{S}\langle\bar{\psi} \tilde{\psi}\rangle}{N_{f}}=b_{1} m+b_{2} m^{3}+\{\text { finite terms }\},
$$

with the asymptotic behaviour of $b_{1}$ and $b_{2}$ being $1 / a^{2}$ and $\ln (a)$ respectively. In finite volume it is useful to define the chiral condensate in fixed topological sectors $\nu=|Q|$. It can be parameterized as

$$
-\frac{\langle\bar{\psi} \tilde{\psi}\rangle_{\nu}}{N_{f}}=\frac{\nu}{V m}+\chi_{\nu}
$$

where

$$
\hat{\chi}_{\nu}=Z_{S} \chi_{\nu}=b_{1} m+b_{2} m^{3}+\{\text { finite terms }\} .
$$

The $1 / m$ infrared divergence in Eq. (2.8) is the trivial topology-dependent contribution due to the presence of the zero modes of $D$ in the quark propagator. The renormalization constant $Z_{S}$, and the additive subtractions, which are needed to remove the additive 
ultraviolet divergences, can be chosen to be independent on the topology, see Appendix B. As a consequence the continuum limit of the combinations

$$
\hat{\chi}_{\nu_{1}}-\hat{\chi}_{\nu_{2}}
$$

is unambiguously defined at finite quark mass provided the volume is large enough.

The spectral density $\rho(\lambda)$ of the massive Dirac operator $D_{m}$ has a well defined thermodynamic limit. It can be defined as [38]

$$
\rho(\lambda)=\frac{1}{V} \sum_{k}\left\langle\delta\left(\lambda-\left|\lambda_{k}\right|\right)\right\rangle
$$

where $\left|\lambda_{k}\right|^{2}$ are the non topological eigenvalues of $D^{\dagger} D$ restricted to one of the chiral sectors. Recently it has been shown that its renormalized counterpart

$$
\hat{\rho}(\lambda)=Z_{S} \rho\left(Z_{S} \lambda\right)
$$

has a universal continuum limit [30]. The same applies to the spectral density $\rho_{\nu}(\lambda)$ defined in the theory at fixed topology. At this point the renormalized spectral density $\hat{\rho}(\lambda)$ can, in principle, be computed with lattice simulations. However it is more realistic from a numerical point of view to consider integrals of $\hat{\rho}(\lambda)$ with a given probe function. There is clearly a lot of choice. In the rest of our paper we will focus on observables "condensate-like" of the form

$$
\hat{\tau}_{\nu}\left(\hat{\lambda}_{\min }, \hat{\lambda}_{\max }\right)=2 \hat{m} \int_{\hat{\lambda}_{\min }}^{\hat{\lambda}_{\max }} \frac{1-\left(\bar{a} Z_{S} \hat{\lambda} / 2\right)^{2}}{\left[1-\left(\bar{a} Z_{S} \hat{m} / 2\right)^{2}\right] \hat{\lambda}^{2}+\hat{m}^{2}} \hat{\rho}_{\nu}(\hat{\lambda}) d \hat{\lambda},
$$

with $\hat{\lambda}=\lambda / Z_{S}$ and $\hat{m}=m / Z_{S}$, which have a well defined continuum limit if $\hat{\lambda}_{\text {min }}$ and $\hat{\lambda}_{\text {max }}$ are kept fixed when $a \rightarrow 0$. For $\hat{\lambda}_{\text {max }} \ll \Lambda_{\mathrm{QCD}}$ these integrals are expected to be dominated from the most infrared part of the Dirac spectrum (see below) where also discretization effects are expected to be quite small. A more detail study of other possible choices is left for future studies. At fixed lattice spacing the quantity

$$
\hat{\tau}_{\nu}\left(\hat{\lambda}_{\min }, \infty\right)=\lim _{\hat{\lambda}_{\max } \rightarrow \infty} \hat{\tau}_{\nu}\left(\hat{\lambda}_{\min }, \hat{\lambda}_{\max }\right)
$$

has the same ultraviolet divergences of the chiral condensate in Eq. (2.9). They arise from the contributions to the integral at the upper end of the integration domain. Since they are topology independent, the observables

$$
\hat{\tau}_{\nu_{1}}\left(\hat{\lambda}_{\min 1}, \infty\right)-\hat{\tau}_{\nu_{2}}\left(\hat{\lambda}_{\min 2}, \infty\right)
$$

are unambiguously defined. The chiral condensate $\hat{\chi}_{\nu}$ is recovered by taking $\hat{\lambda}_{\text {min }}=0$ in Eq. (2.14). The coefficient of the leading order in $\hat{m}$ is the first Leutwyler-Smilga sum rule $[17]$

$$
\left.\frac{1}{V} \frac{\partial}{\partial \hat{m}} \hat{\chi}_{\nu}\right|_{m=0}=\frac{2}{V} \int_{0}^{\infty} \frac{1-\left(\bar{a} Z_{S} \hat{\lambda} / 2\right)^{2}}{\hat{\lambda}^{2}} \hat{\rho}_{\nu}(\hat{\lambda}) d \hat{\lambda} .
$$

and differences of sum rules analogous to Eq. (2.10) are free from ultraviolet ambiguities. All these considerations remain valid in the so-called quenched approximation, where the fermion determinant is dropped in the effective gluon action. 


\section{The quark condensate in the effective chiral theory}

In presence of spontaneous symmetry breaking, the QCD correlation functions at small masses and momenta are expected to match those of a chiral effective theory which contains only the pseudo-Goldstone bosons as dynamical degrees of freedom [1,2]. At asymptotically large volumes $L \gg 1 / \Lambda_{\mathrm{QCD}}$ and for small quark masses the finite volume behaviour of QCD correlation functions can then be predicted [39,40]. In the mass range $\hat{m} \Sigma V \lesssim 1$ the partition function of the effective theory reads

$$
\mathcal{Z}=\int_{S U\left(N_{f}\right)} d U_{0} \exp \left[\mu \operatorname{Re} \operatorname{Tr} U_{0}\right]
$$

where $U_{0}$ are the zero-momentum modes in the chiral theory, $d U_{0}$ is the corresponding Haar measure, $\mu=\hat{m} \Sigma V$, and $\Sigma$ is the infinite volume chiral condensate. The quark condensate (with opposite sign) is given by [17]

$$
\Sigma(\mu)=\frac{\Sigma}{N_{f}} \frac{\partial}{\partial \mu} \ln \mathcal{Z}=\frac{\Sigma}{2 N_{f}} \mu \cdot\left\{\begin{array}{ll}
2 & N_{f}=2 \\
1 & N_{f} \geq 3
\end{array}+\cdots,\right.
$$

and it vanishes in the chiral limit since spontaneous symmetries breaking can only occur in infinite volume. For $\mu \ll 1$ the linear proportionality of the condensate to the quark mass and the volume is a distinctive sign of a theory which undergoes spontaneous symmetry breaking in infinite volume $[16,17,38]$. It is due to the long-range correlations left in the system at finite volume. The quark condensate plays the rôle of the magnetization of a ferromagnet with the quark mass being the analogous of the external magnetic field. A tiny variation of the quark mass requires a variation of order $(\hat{m} V)$ in the condensate. This behaviour is not spoiled in QCD at finite lattice spacing if fermions preserve an exact chiral symmetry. The mass-dependent ultraviolet divergences in Eq. (2.7) affect only the sub-leading corrections suppressed by a factor $1 / V$.

The partition function of the theory at fixed topological charge $\nu$ at asymptotically large volumes and in the same mass range reads

$$
\mathcal{Z}_{\nu}=\int_{U\left(N_{f}\right)} d U(\operatorname{det} U)^{\nu} \exp [\mu \operatorname{Re} \operatorname{Tr} U]
$$

and the quark condensate is given by [17]

$$
\Sigma_{\nu}(\mu)=\frac{\Sigma \nu}{\mu}+\tilde{\chi}_{\nu}(\mu)=\frac{\Sigma}{N_{f}} \frac{\partial}{\partial \mu} \ln \mathcal{Z}_{\nu}=\frac{\Sigma \nu}{\mu}+\frac{\Sigma}{2\left(N_{f}+\nu\right)} \mu+\cdots \quad N_{f} \geq 2
$$

The infrared divergence matches exactly the analogous one on the QCD side in Eq. (2.8). For $\mu \ll 1$ the linear proportionality of the condensate to the quark mass and the volume signals in this case the presence of a non-zero condensate in the thermodynamic limit of the full theory. The low-energy constant $\Sigma$ can be determined with arbitrary precision, if the quark mass and the volume $V$ are properly tuned, by matching the combinations

$$
\tilde{\chi}_{\nu_{1}}(\mu)-\tilde{\chi}_{\nu_{2}}(\mu)
$$


with the QCD counterparts in Eq. (2.10). The next-to-leading (NLO) corrections to the scaling behaviour can be computed in so-called $\epsilon$-expansion [40]. They are suppressed as $(4 \pi F L)^{-2}$, with $F$ being the Goldstone boson decay constant in the chiral limit, and they can be included by replacing $\Sigma$ with the volume dependent parameter $\Sigma_{\text {eff }}(V)$, whose expression can be found in Ref. [40]. This implies that the ratios

$$
\left.\frac{\tilde{\chi}_{\nu_{1}}(\mu)-\tilde{\chi}_{\nu_{2}}(\mu)}{\tilde{\chi}_{\nu_{3}}(\mu)-\tilde{\chi}_{\nu_{4}}(\mu)}\right|_{\mu=0}=\frac{\left(\nu_{1}-\nu_{2}\right)}{\left(\nu_{3}-\nu_{4}\right)} \frac{\left(\nu_{3}+N_{f}\right)\left(\nu_{4}+N_{f}\right)}{\left(\nu_{1}+N_{f}\right)\left(\nu_{2}+N_{f}\right)}, \quad \nu_{3} \neq \nu_{4}
$$

are non-trivial parameter free predictions of the effective theory up to NLO, which is the higher order at which sub-leading corrections are presently known. The functional form of the condensate $\Sigma_{\nu}(\mu)$ is known for arbitrary values of $\mu \leq 1[17,41]$. The condensate is also known in a partially quenched chiral effective theory with $N_{f}$ flavors and an extra valence one of mass $\hat{m}_{v}$. From its discontinuity across the imaginary axis

$$
\left.\operatorname{Disc}\right|_{\hat{m}_{v}=i \hat{\lambda}} \sum_{\nu}^{\mathrm{pQ}}\left(\mu, \hat{m}_{v}\right)=\lim _{\epsilon \rightarrow 0} \Sigma_{\nu}^{\mathrm{pQ}}(\mu, i \hat{\lambda}+\epsilon)-\Sigma_{\nu}^{\mathrm{pQ}}(\mu, i \hat{\lambda}+\epsilon)=2 \pi \tilde{\rho}_{\nu}(\hat{\lambda})
$$

it is possible to extract the NLO effective theory prediction $\tilde{\rho}(\hat{\lambda})$ of the spectral density in presence of $N_{f}$ flavors [21,22]. This is the functional form that is expected to match $\hat{\rho}(\hat{\lambda})$ in Eq. (2.12) for $\hat{\lambda} \ll \Lambda_{\mathrm{QCD}} \ll 1 / \bar{a}$. In the following for brevity we report the full expressions for the chiral condensate and the spectral density for the quenched case only, which is the one needed in rest of the paper.

In the quenched approximation of QCD, an effective low-energy chiral theory is formally obtained if an additional expansion in $1 / N_{c}$, where $N_{c}$ is the number of colors, is carried out together with the usual one in quark masses and momenta [42,43]. Here we adopt the pragmatic assumption that the quenched chiral theory describes the lowenergy regime of quenched QCD in certain ranges of kinematic scales at fixed $N_{c}$. Correlation functions can be parameterized in terms of effective coupling constants, the latter being defined as the couplings of the effective theory. The quenched condensate at fixed topology $\nu$ at asymptotically large volumes (LO) is given by $[21,22]$

$$
\frac{\Sigma_{\nu}^{\mathrm{LO}}(\mu)}{\Sigma}=\mu\left(I_{\nu}(\mu) K_{\nu}(\mu)+I_{\nu+1}(\mu) K_{\nu-1}(\mu)\right)+\frac{\nu}{\mu},
$$

where $I_{\nu}, K_{\nu}$ are modified Bessel functions. At the NLO in the $\epsilon$-expansion the condensate is $[21,44]$

$$
\mu \Sigma_{\nu}^{\mathrm{NLO}}(\mu)=\mu_{\mathrm{eff}} \Sigma_{\nu}^{\mathrm{LO}}\left(\mu_{\mathrm{eff}}\right),
$$

with $\mu_{\mathrm{eff}}=m \Sigma_{\mathrm{eff}} V$. The infrared sickness of quenched chiral perturbation theory, as well as of quenched QCD, manifests itself in the fact that $\Sigma_{\text {eff }}$ diverges logarithmically with $L$. In the following we define operatively $\Sigma_{\text {eff }}$ at a given volume as

$$
\left.\frac{2 \nu \tilde{\chi}_{\nu}(\mu)}{\hat{m} V}\right|_{\hat{m}=0}=\Sigma_{\text {eff }}^{2}(V) \quad \nu>0 \text {. }
$$



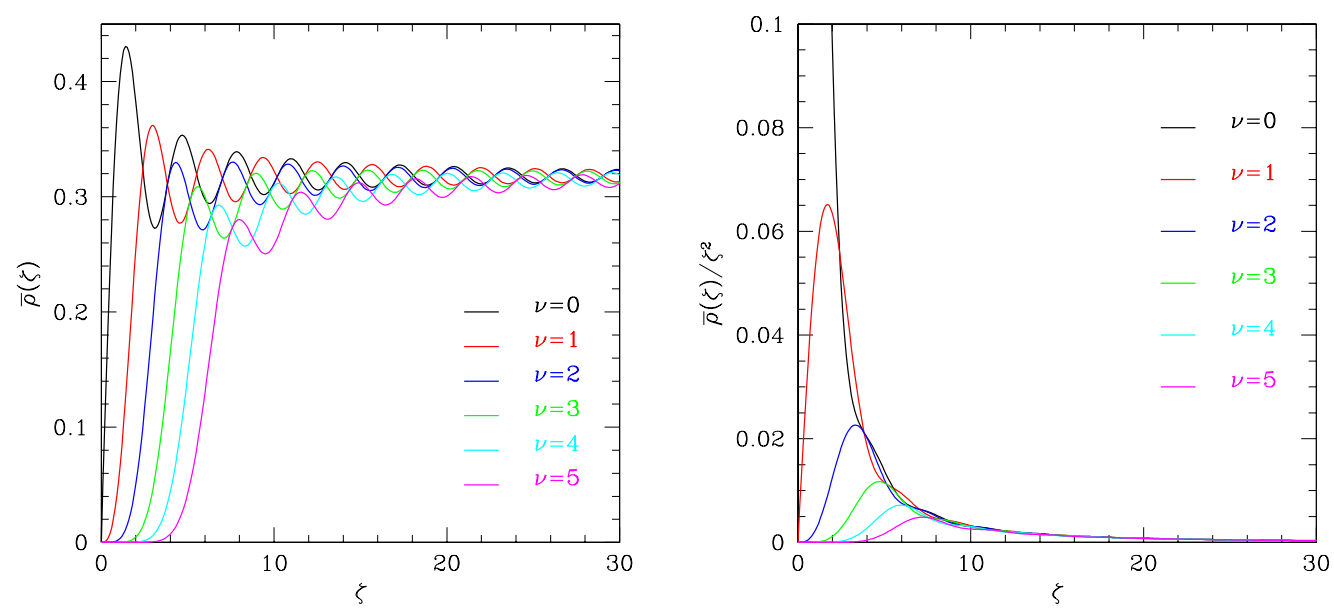

Figure 1: The microscopic spectral density and $\bar{\rho}_{\nu}(\zeta) / \zeta^{2}$ for $\nu=0-5$ are shown on the left and on the right plots respectively.

If we consider two box sizes $V_{1,2}=L_{1,2}^{4}$, the volume dependence of $\Sigma_{\text {eff }}$ at NLO in $\epsilon$-expansion of the quenched chiral theory is $[44,45]$

$$
\frac{\Sigma_{\text {eff }}\left(V_{1}\right)}{\Sigma_{\text {eff }}\left(V_{2}\right)}=1+\frac{1}{3 F^{2}}\left\{\frac{m_{0}^{2}}{8 \pi^{2}} \ln \left(\frac{L_{1}}{L_{2}}\right)-\beta_{1} \alpha\left(\frac{1}{L_{1}^{2}}-\frac{1}{L_{2}^{2}}\right)\right\}
$$

where $\beta_{1}=0.140461$ is a shape coefficient [46], $m_{0}$ and $\alpha$ are the singlet mass and the additional low-energy constant of the singlet kinetic term in the chiral Lagrangian respectively (see Ref. [44] for unexplained notation). From the discussion above it follows that the ratios in Eq. (3.6), for $\nu_{i}>0$ and with $N_{f}=0$, are non-trivial parameterfree predictions also in the quenched effective theory up to the sub-leading corrections of higher order presently known. The spectral density of the Dirac operator in the quenched approximation can be extracted from Eqs. (3.7), (3.8) and (3.9). They lead to the microscopic spectral density $[21,44]$

$$
\bar{\rho}_{\nu}(\zeta)=\frac{\zeta}{2}\left[J_{\nu}(\zeta)^{2}-J_{\nu+1}(\zeta) J_{\nu-1}(\zeta)\right]
$$

which is related to $\tilde{\rho}_{\nu}$ as

$$
\bar{\rho}_{\nu}(\zeta)=\frac{1}{\Sigma} \tilde{\rho}_{\nu}(\zeta / \Sigma V)
$$

with $\zeta=\hat{\lambda} \Sigma V$. For $\hat{\lambda} \ll \Lambda_{\mathrm{QCD}}$ the integral $\hat{\tau}_{\nu}\left(\hat{\lambda}_{\min }, \hat{\lambda}_{\max }\right)$ in Eq. (2.13) can then be matched with

$$
\tilde{\tau}_{\nu}\left(\frac{\zeta_{\min }}{\Sigma V}, \frac{\zeta_{\max }}{\Sigma V}\right)=2 \Sigma \mu \int_{\zeta_{\min }}^{\zeta_{\max }} \frac{1}{\zeta^{2}+\mu^{2}} \bar{\rho}_{\nu}(\zeta) d \zeta
$$


The functions $\bar{\rho}_{\nu}(\zeta)$ and $\bar{\rho}_{\nu}(\zeta) / \zeta^{2}$ are shown in Fig. 1. They highlights the fact that large parts of the integrals in Eq. (3.14) come from the region with $\zeta \lesssim 20$, where a handful of low-lying eigenvalues of the Dirac operator provides the bulk of the contribution. Similar conclusions apply in the range $\mu \leq 1$.

\section{Numerical computation}

The primary observables in our numerical computations are the integrals $\hat{\tau}_{\nu}\left(\hat{\lambda}_{\text {min }}, \infty\right)$ defined in Eq. (2.14). The lower bound $\hat{\lambda}_{\text {min }}$ is chosen to ensure the reliability of the Monte Carlo estimate (see below) and at the same time to minimize the difference with $\hat{\chi}_{\nu}$. The low energy constant $\Sigma_{\text {eff }}$ is extracted from a matching of the combinations in Eq. (2.15) with the corresponding formulas in the effective theory. Our final goal, the computation of $\left(\hat{\chi}_{\nu_{1}}-\hat{\chi}_{\nu_{2}}\right)$, is then achieved by adding the small contribution from the infrared tail analytically using the functional form in Eq. (3.14).

\subsection{Numerical estimator}

The underlying chiral symmetry guarantees that for every background gauge configuration the quark propagator $S_{m}(x, y)$ (see Appendix A) satisfies

$$
\sum_{x} \operatorname{tr}\left[S_{m}(x, x)\right]=\frac{\nu}{m}+2 \sum_{x} \operatorname{tr}\left[P_{c} S_{m}(x, x) P_{c}\right]
$$

with $\nu$ being the absolute value of the topological charge of the configuration and $P_{c}$ the chiral projector into the chiral sector without zero modes 2 . In this form, and once the topological charge is known, the calculation of the quark condensate requires the computation of the propagator in the sector without zero modes only [7].

On reasonably large lattices the computation of all eigenvalues of the Dirac operator or of the propagator from every source point $x$ is numerically unfeasible. It is quite standard, however, to compute the quark propagator from a few fixed source points or to extract a few low-lying eigenvectors. Let us assume that once $\nu$ has been determined, a number $n$ of approximate low-lying eigenvalues of $P_{c} D^{\dagger} D P_{c}$ and the corresponding eigenvectors is computed by minimizing the Ritz functional starting from random vectors generated with a Gaussian action, i.e. invariant under space-time translations [47]. The minimization is carried out until the approximated eigenvalues have a relative error $\omega_{k}$ and satisfy

$$
\begin{aligned}
P_{c} D^{\dagger} D P_{c} u_{k} & =\left|\bar{\lambda}_{k}\right|^{2} u_{k}+r_{k}, \quad(k=1, \ldots, n) ; \\
\left(u_{l}, u_{k}\right) & =\delta_{l k} ; \\
\left(u_{l}, r_{k}\right) & =0 \forall(l, k), \quad\left\|r_{k}\right\| \leq \omega_{k}\left|\bar{\lambda}_{k}\right|^{2} .
\end{aligned}
$$

\footnotetext{
${ }^{2}$ In finite volume the probability of having a configuration with zero modes in both chiralities is zero.
} 
The propagator in the sector without zero modes can then be split in a light and a heavy contribution as follows:

$$
P_{c} S_{m}(x, y) P_{c}=m \sum_{k=1}^{n} \frac{P_{c} \tilde{u}_{k}(x) u_{k}^{\dagger}(y) P_{c}}{\left(1-\bar{a}^{2} m^{2} / 4\right)\left|\bar{\lambda}_{k}\right|^{2}+m^{2}}+P_{c} S^{h}(x, y) P_{c},
$$

where $\tilde{u}_{k}$ is defined from $u_{k}$ as in Eq. (A.9). It is easy to prove that, once averaged over the gauge configurations, the spin-color trace of each contribution on the right-hand side of Eq. (4.5) is translational invariant even if the $u_{k}$ are only approximate eigenvectors, i.e. $\omega_{k} \neq 0$ [47]. The condensate, after the trivial contribution from the zero modes is subtracted, can then be decomposed as

$$
\chi_{\nu}=\frac{1}{V} \sum_{x}\left\langle\operatorname{tr}\left[S_{m}(x, x)\right]\right\rangle_{\nu}-\frac{\nu}{m V}=\chi_{\nu}^{l}+\chi_{\nu}^{h},
$$

where the heavy and the light contributions can be computed as

$$
\begin{aligned}
\chi_{\nu}^{l} & =\frac{2 m}{V} \sum_{k=1}^{n}\left\langle\frac{1-\bar{a}^{2}\left|\bar{\lambda}_{k}\right|^{2} / 4}{\left(1-\bar{a}^{2} m^{2} / 4\right)\left|\bar{\lambda}_{k}\right|^{2}+m^{2}}\right\rangle_{\nu}, \\
\chi_{\nu}^{h} & =2\left\langle\operatorname{tr}\left[P_{c} S^{h}(0,0) P_{c}\right]\right\rangle_{\nu} .
\end{aligned}
$$

It must be stressed that Eq. (4.6) is exact independently on the values of $\omega_{k}$ and the number of the extracted eigenvectors $n$. By contrast, the statistical variance of the estimator changes with $\omega_{k}$ and $n$. The local fluctuations of the approximated eigenvectors $u_{k}(x)$ are enhanced on the r.h.s. of Eq. (4.5) by the smallness of the denominator. Under the working assumption that they are responsible for large variations in the trace of the local propagator, the variance of the estimator in Eq. (4.6) is greatly reduced with respect to the one of $\operatorname{tr}\left[P_{c} S(0,0) P_{c}\right]$. Deep in the chiral regime the probability of having a configuration with eigenvalues lying in the infrared tail of the spectral density $\hat{\rho}(\lambda)$ is small but not negligible. The tail is poorly sampled by the Monte Carlo while at the same time the smallness of the eigenvalues generates large fluctuations in the light part of the condensate $\chi_{\nu}^{l}$. To overcame this problem we replace $\chi_{\nu}^{l}$ with

$$
\tau_{\nu}^{l}=\frac{2 m}{V} \sum_{k=1}^{n}\left\langle\frac{1-\bar{a}^{2}\left|\bar{\lambda}_{k}\right|^{2} / 4}{\left(1-\bar{a}^{2} m^{2} / 4\right)\left|\bar{\lambda}_{k}\right|^{2}+m^{2}} \theta\left(\left|\lambda_{k}\right|-\lambda_{\min }\right)\right\rangle_{\nu},
$$

in Eq. (4.6), where $\theta$ is the usual step function. The value of $\lambda_{\min }$ is chosen in such a way that only 5 eigenvalues in all the Monte Carlo history of a given data set are lower. The parameter $\Sigma_{\text {eff }}$ is then extracted by matching these observables with the analogous ones in the effective theory. Eventually the chiral condensate $\chi_{\nu}$ is computed by adding the small contribution from the tail analytically using the formula in Eq. (3.14). When the latter is substantial the determination of $\Sigma_{\text {eff }}$ from differences of $\hat{\tau}_{\nu}\left(\hat{\lambda}_{\text {min }}, \infty\right)$ is still correct, but the value of $\chi_{\nu}$ is heavily affected by the functional form used and therefore 


\begin{tabular}{|c|cccc|c|c|}
\hline lat & $\beta$ & $L / a$ & $L(\mathrm{fm})$ & $N_{\mathrm{cfg}}$ & $N_{\mathrm{cfg}}^{\nu}$ & $a m$ \\
\hline c1 & 5.8458 & 12 & $1.49 \mathrm{fm}$ & 672 & $119,205,155$, & $0.001,0.003,0.008$, \\
& & & & & $104,51,29$ & $0.012,0.0016$ \\
\hline c2 & 5.8458 & 16 & $1.98 \mathrm{fm}$ & 488 & $49,69,82$, & $0.000316,0.000949,0.00253$, \\
& & & & & $72,50,54$ & $0.00380,0.00506$ \\
\hline c3 & 6.0 & 16 & $1.49 \mathrm{fm}$ & 418 & $74,137,101$, & $0.000612,0.00184,0.00490$, \\
& & & & & $62,27,12$ & $0.00735,0.00980$ \\
\hline
\end{tabular}

Table 1: Parameters of the simulations: $\beta=6 / g^{2}$ is the bare gauge coupling, $L$ is the linear extent of the each lattice, $N_{\text {cfg }}$ is the total number of configurations generated, and $a m$ are the bare quark masses considered. $N_{\mathrm{cfg}}^{\nu}$ refers to the subset of configurations with fixed topological charge for $\nu=0-5$.

it less interesting to us. In the following we only consider data sets where the integral of the tail is at most 25 per cent of $\tilde{\chi}_{\nu}$, and in most of the cases it is less than 10 per cent. A positive side effect of this requirement is that the computation of the eigenvalues does not have to be very precise. The tail contribution in $\chi_{\nu}$ can, of course, be reduced by increasing the statistics of the data set, i.e. sampling part of the tail with confidence.

This procedure complements for the case of the chiral condensate the low-mode averaging (LMA) technique proposed in Ref. [48]. The latter has been successfully applied already to meson two-point functions [48-50], baryon two-point functions [51] and more recently to three-point functions for the extraction of low-energy constants of the $\Delta S=1$ chiral effective Hamiltonian [52].

\subsection{Numerical experience}

We have generated ensembles of gauge configurations by standard Monte Carlo techniques with the Wilson gluon action and periodic boundary conditions. The fermions are discretized with the Neuberger-Dirac operator as defined in Eq. (A.3) with $s=0.4$. A summary of the parameters of our runs are reported in Tab. 1. We have simulated three lattices with two different volumes (runs c1 and c2) and two lattice spacings (runs c1 and c3). The linear extent always satisfies $L \gtrsim 1.5 \mathrm{fm}$, a size that we expect to be large enough for a finite-size scaling study. This is suggested by the results in Ref. [12], where for $L \gtrsim 1.5 \mathrm{fm}$ it was found a detailed agreement of the predictions of random matrix theory for the low-lying eigenvalues of the Dirac operator with quenched QCD results. The values of the two lattice spacings are chosen to guarantee the locality of the Neuberger operator [53], and to be in a range where discretization effects were found to be small in several observables $[13,54]$. The quark masses for the lattice $\mathrm{c} 1$ are fixed to be roughly in the interval $0.07 \lesssim \mu \lesssim 1.2$. For the lattice $\mathrm{c} 2$ the masses are such that the values of $(m V)$ match those of $\mathrm{c} 1$. The masses for the lattice $\mathrm{c} 3$ are chosen so that the dimensionless quantity $\left(m V / \hat{Z}_{S} r_{0}^{3}\right)$ is constant, where $r_{0}=0.5 \mathrm{fm}$ is a widely used reference scale 

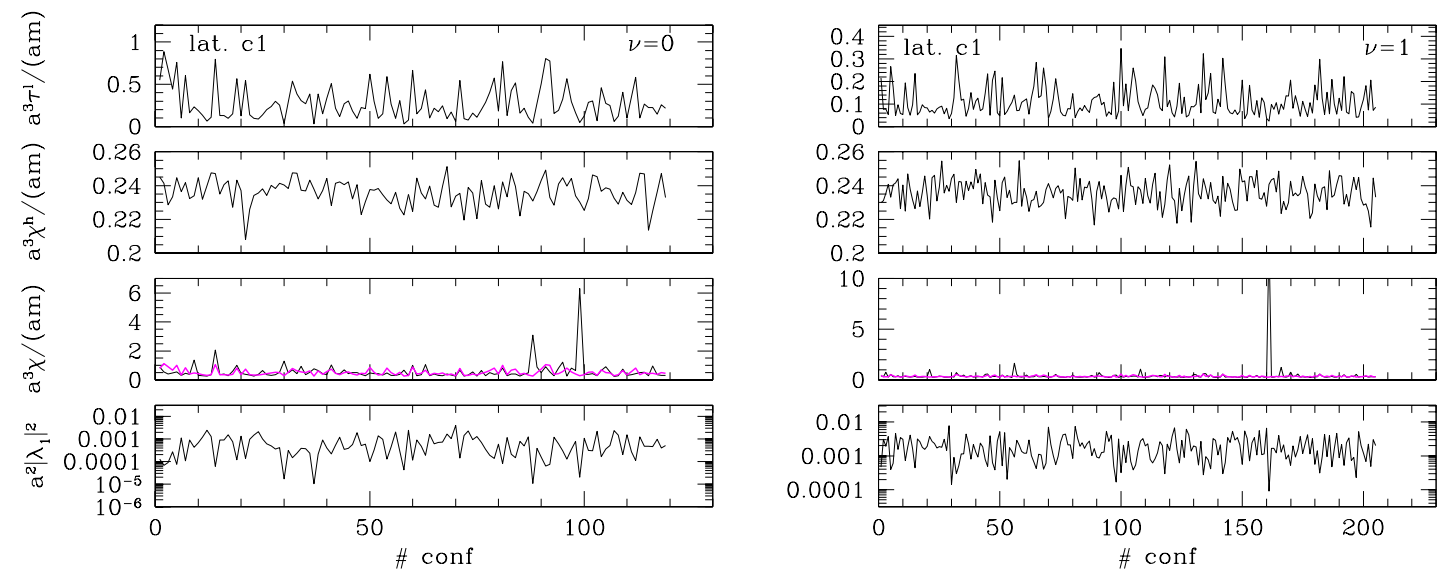

Figure 2: Monte Carlo history for the run $\mathrm{c} 1, \mathrm{am}=0.008$ and for the topological sectors $\nu=0$ (left) and $\nu=1$ (right). The first plot from the bottom shows the Monte Carlo history of the absolute value of the smallest eigenvalue of $P_{c} D^{\dagger} D P_{c}$; the second one represents the condensate (divided by the quark mass) computed without LMA (blackthin line) and the sum $a^{3}\left(\tau_{\nu}^{l}+\chi_{\nu}^{h}\right) /(a m)$ (magenta-thick line). The third and the fourth show the Monte Carlo histories of the heavy contribution $\left(a^{3} \chi_{\nu}^{h}\right) / a m$ and the light one $\left(a^{3} \tau_{\nu}^{l}\right) / a m$, respectively.

in quenched QCD computations $[55,56]$ and $\hat{Z}_{S}$ is the renormalization constant of the scalar density in the RGI scheme which we have taken from Ref. [13]. The calculation of the topological charge, of the low-lying eigenvalues, and of the quark propagator is performed following Ref. [47]. For each run the low-mode averaging is implemented as described in the previous subsection with $n=20$ and $\omega_{k}=0.05$. A posteriori we have verified that the highest eigenvalue extracted satisfies $\left(\left\langle\left|\bar{\lambda}_{20}\right|\right\rangle \Sigma_{\text {eff }} V\right)>20$ for all lattices.

In Fig. 2 we show a typical Monte Carlo history for the smallest eigenvalue of the Neuberger operator, for the chiral condensate with and without LMA and for the heavy and light contributions separately. It is obtained from the run c1 for $\nu=0,1$ and $a m=0.008$. A first observation is that the heavy part of the condensate is very stable in all topological sectors. Moreover the quantity $\operatorname{tr}\left[P_{c} S^{h}(0,0) P_{c}\right] /(a m)$ is essentially independent on the quark mass configuration by configuration. For all lattices, its largest relative deviation that we have observed among different masses is roughly $10^{-3}$. We interpret this as a consequence of the fact that $\left|\bar{\lambda}_{20}\right| \gg m$ configuration by configuration. We thus expect that a further stabilization of the heavy contribution would not reduce the variance of the condensate significantly. The Monte Carlo history of $\tau_{\nu}^{l}$ does not show large spikes for all masses, and a statistical analysis is applicable. Some of the lighter masses at lower topologies, however, have been discarded (see Tab. 21) to satisfy the upper limit on the tail contribution discussed in the previous subsection. As expected the light part fluctuates much more than the heavy one. Large contributions 
appear in coincidence with the lower values of $\left|\lambda_{1}^{2}\right|$ consistently with the expectations. The Monte Carlo history of the local estimator $\operatorname{tr}\left[P_{c} S(0,0) P_{c}\right]$ has fluctuations which are much larger than those with LMA. Moreover for the lower topologies extreme statistical fluctuations are observed for most of the masses considered, which invalidate the statistical analysis of the sample. In the following the physics analysis is carried out only on the data with LMA.
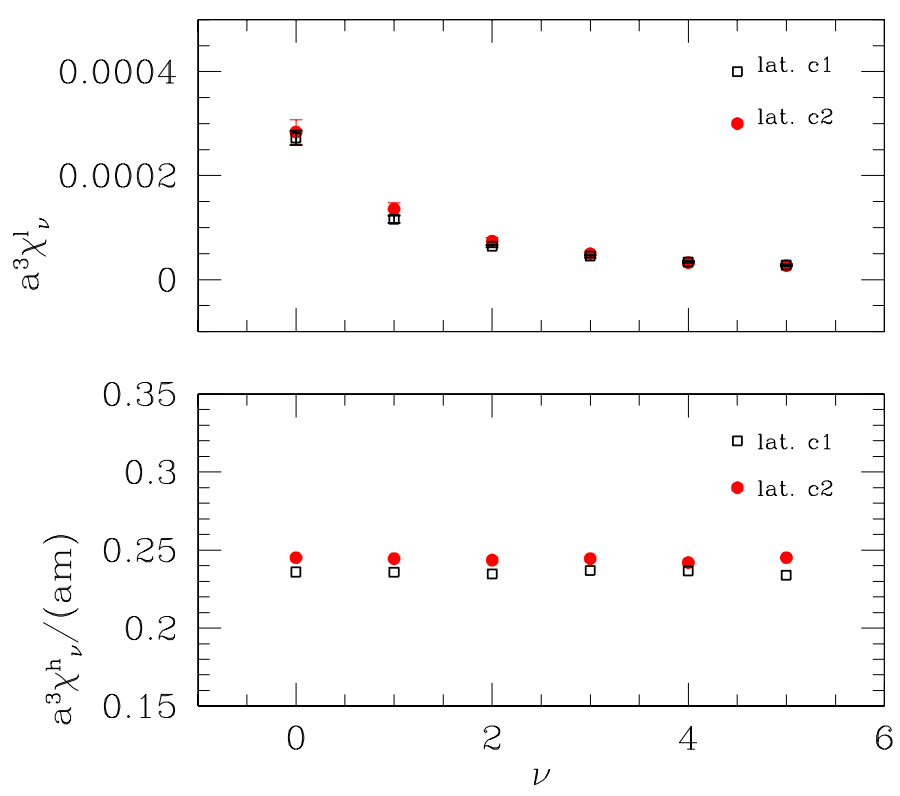

Figure 3: The quantities $a^{2} \chi_{\nu}^{h} / m$ (bottom) and $a^{3} \chi_{\nu}^{l}$ (top) as a function of $\nu$, for the second heaviest mass of the run c1 (black points) and run c2 (red points).

In Fig. 3 we show our results for the heavy and the light contributions to the chiral condensate as a function of $\nu$ for the second heaviest mass of the runs $\mathrm{c} 1$ and $\mathrm{c} 2$. The heavy part divided by the quark mass is weakly dependent on the topological charge and the volume. The results for $a^{3} \chi_{\nu}^{l}$ at the corresponding masses are compatible. This indicates that the light contribution is to a good approximation a function of the variable $(m V)$. In addition, the $1 / \nu$ behavior predicted close to the chiral limit by the first Leutwyler-Smilga sum rule is reproduced qualitatively. These observations, which are valid for all masses simulated, point to the fact that the splitting between heavy and light contribution is such that the bulk of the heavy part behaves essentially like an ultraviolet divergence (i.e. volume and topology independent and linear in the mass), while the light part scales with respect to topology, mass and volume essentially as predicted by chiral perturbation theory. The analysis in the chiral effective theory in 

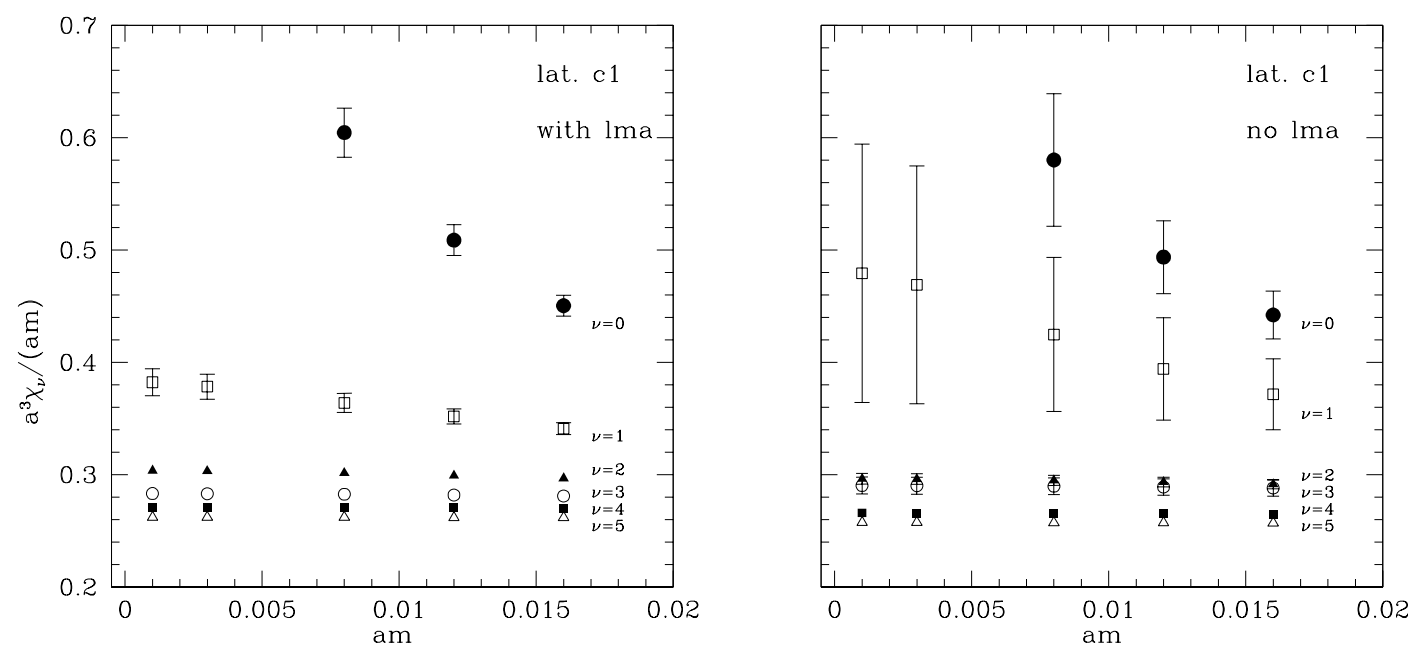

Figure 4: Results for $\left(a^{3} \chi_{\nu}\right) / a m$ as a function of the quark mass, for the lattice $\mathrm{c} 1$ at several values of $\nu$. The data on the left are obtained with low-mode averaging, while on the right we show the values computed with the local estimator.

the previous section suggests that these features are mostly volume independent.

The effect of low-mode averaging can be appreciated in Fig. 4, where we show $\left(a^{3} \chi_{\nu}\right) / a m$ for $\nu=0-5$ as a function of the quark mass, with (left) and without (right) LMA 3 . It is clear that the variance reduction is much more effective for the sectors with lower topological charge, which are dominated by infrared contributions. Nevertheless with LMA we still obtain a variance reduction of a factor $\sim 2$ up to $\nu=5$ in all our runs.

\section{Comparison with the effective theory}

In this section we compare our numerical results with the predictions of the chiral effective theory. We first focus on the finite-size scaling and the topology dependence of the condensate, and then we extract the value of $\Sigma_{\text {eff }}$ from a fit in the mass of our data. Our raw numerical results of $\chi_{\nu}$ for $\nu=0-5$, are reported in Table 2. The statistical errors have been estimated with a jackknife procedure.

\subsection{Finite-size scaling}

The parameters of the lattices c1 and c2 have been chosen to carry out a finite-size scaling study. The lattice spacing is the same and the physical volume differ by more than a factor 3 . The bare combinations $a^{3}\left(\chi_{1}-\chi_{2}\right) / a m$ and $a^{3}\left(\chi_{2}-\chi_{3}\right) / a m$ computed

\footnotetext{
${ }^{3}$ At lower topologies and without LMA the average values and their errors are only indicative since the statistical analysis is invalidated by extreme statistical fluctuations.
} 


\begin{tabular}{|l|l|ccccc|}
\hline lat & $\nu \backslash a m$ & 0.001 & 0.003 & 0.008 & 0.012 & 0.016 \\
\hline c1 & 0 & & & $0.00484(18)$ & $0.00610(16)$ & $0.00721(15)$ \\
& 1 & $0.000382(12)$ & $0.00114(3)$ & $0.00291(7)$ & $0.00422(8)$ & $0.00546(8)$ \\
& 2 & $0.000304(3)$ & $0.000910(8)$ & $0.002413(19)$ & $0.00359(3)$ & $0.00475(3)$ \\
& 3 & $0.000283(3)$ & $0.000849(8)$ & $0.002261(21)$ & $0.00338(3)$ & $0.00450(4)$ \\
& 4 & $0.0002712(22)$ & $0.000814(7)$ & $0.002168(17)$ & $0.00325(3)$ & $0.00433(3)$ \\
& 5 & $0.0002622(23)$ & $0.000787(7)$ & $0.002097(18)$ & $0.00314(3)$ & $0.00419(4)$ \\
\hline \hline lat & $\nu \backslash a m$ & 0.000316 & 0.000949 & 0.00253 & 0.00380 & 0.00506 \\
\hline c2 & 0 & & & & $0.0043(3)$ & $0.00480(25)$ \\
& 1 & & & $0.00182(12)$ & $0.00256(14)$ & $0.00319(15)$ \\
& 2 & $0.000157(9)$ & $0.00047(3)$ & $0.00123(6)$ & $0.00182(9)$ & $0.00237(10)$ \\
& 3 & $0.000129(3)$ & $0.000387(8)$ & $0.001026(20)$ & $0.00153(3)$ & $0.00202(4)$ \\
& 4 & $0.000110(4)$ & $0.000329(11)$ & $0.00088(3)$ & $0.00131(4)$ & $0.00174(5)$ \\
& 5 & $0.0001047(21)$ & $0.000314(6)$ & $0.000837(16)$ & $0.001255(24)$ & $0.00167(3)$ \\
\hline \hline lat & $\nu \backslash a m$ & 0.000612 & 0.00184 & 0.00490 & 0.00735 & 0.00980 \\
\hline c3 & 0 & & & $0.00246(11)$ & $0.00324(11)$ & $0.000394(10)$ \\
& 1 & $0.000217(7)$ & $0.000648(19)$ & $0.00168(4)$ & $0.00246(5)$ & $0.00321(5)$ \\
& 2 & $0.0001850(14)$ & $0.000556(4)$ & $0.001474(11)$ & $0.002200(15)$ & $0.002915(19)$ \\
& 3 & $0.0001729(18)$ & $0.000520(5)$ & $0.001382(14)$ & $0.002070(20)$ & $0.00276(3)$ \\
& 4 & $0.0001677(17)$ & $0.000504(5)$ & $0.001342(13)$ & $0.002012(20)$ & $0.00268(3)$ \\
& 5 & $0.0001622(17)$ & $0.000488(5)$ & $0.001299(13)$ & $0.001947(20)$ & $0.00260(3)$ \\
\hline
\end{tabular}

Table 2: Numerical results for $a^{3} \chi_{\nu}$ with $\nu=0-5$.

on these lattices are shown in the plots on the left of Fig. 5. Their values differ from zero by many standard deviations for both volumes. Finite size effects are clearly visible. The rescaled combinations are shown in the plots on the right of the same Figure as a function of $(m V) a^{-3}$. The corresponding data sets are in very good agreement for all masses simulated within the statistical errors. These results are compatible with the finite size scaling behaviour expected at asymptotically large volumes for a theory which exhibits spontaneous symmetry breaking. Within our statistical errors we do not observe deviations from the leading scaling behaviour. Similar conclusions apply for the other combinations made with $\nu=0-3$. Results from higher topological sectors tend to depart from the LO scaling behaviour, but our statistical errors are too large to draw any definite conclusion.

\subsection{First Leutwyler-Smilga sum rule}

The sensitivity on the topology of the spectral density of the low-lying eigenvalues induces a non-trivial $\nu$-dependence in the chiral condensate. The latter has to be compared 

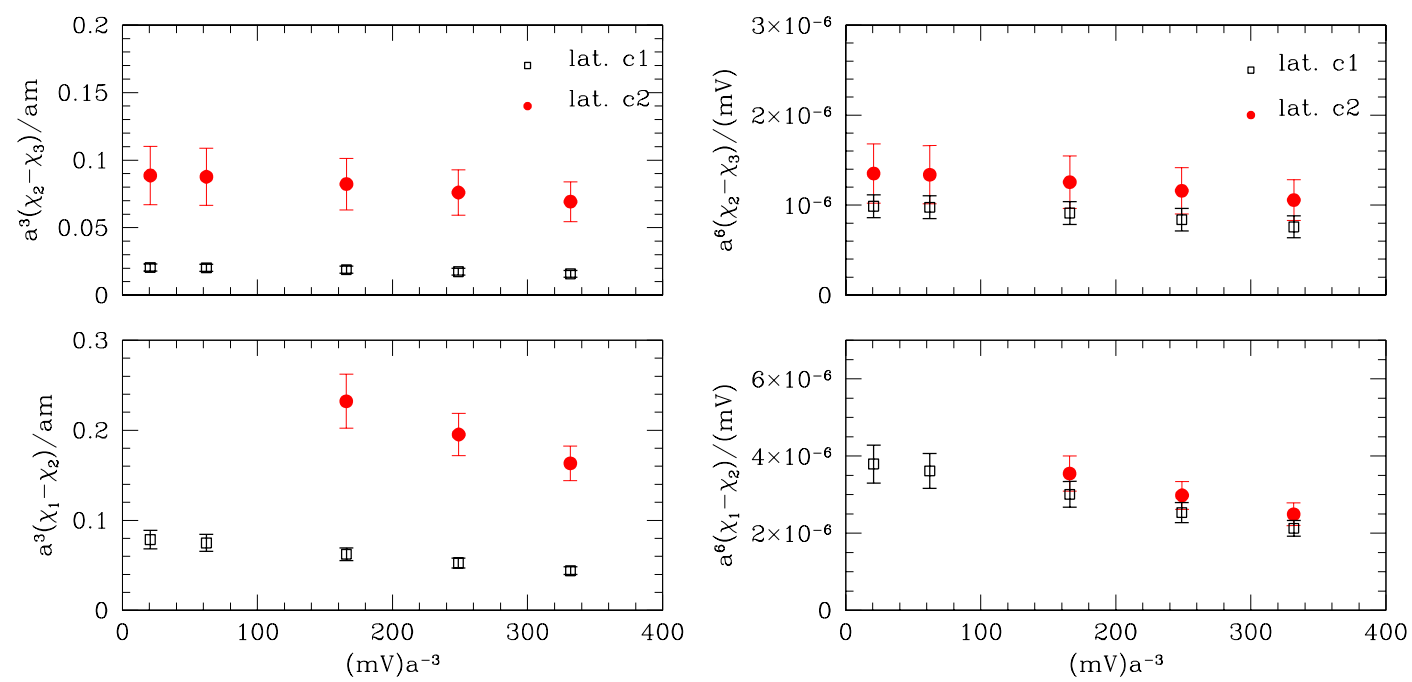

Figure 5: The ratios $a^{3}\left(\chi_{1}-\chi_{2}\right) /(a m)$ and $a^{3}\left(\chi_{2}-\chi_{3}\right) /(a m)$ (left), and $a^{6}\left(\chi_{1}-\chi_{2}\right) /(m V)$ and $a^{6}\left(\chi_{2}-\chi_{3}\right) /(m V)$ (right) as a function of $(m V) a^{-3}$ for lattices $c 1$ and $c 2$.

with the prediction of the chiral effective theory in Eq. (3.8). For the ratios

$$
\frac{\chi_{\nu_{1}}-\chi_{\nu_{2}}}{\chi_{\nu_{3}}-\chi_{\nu_{4}}}
$$

the prediction in the chiral limit is given in Eq. (3.6). It is parameter-free and valid up to the NLO. For the lattice $\mathrm{c} 1$ and $\nu=1-5$, the ratios in Eq. (5.1) are shown in the first plot of Fig. 6 as a function of $(m V) a^{-3}$. As expected from Eq. (3.8) the mass dependence is very mild, and the two lightest points are consistent with a flat behaviour for all combinations. Results for lattices $\mathrm{c} 2$ and $\mathrm{c} 3$ show analogous features. In the plot on the right we report the points at the lightest mass available for each of the three lattices, together with the theoretical expectations from the first Leutwyler-Smilga sum rule in Eq. (3.6). The very good agreement supports the fact that the topology dependence of these ratios is well reproduced in the (quenched) chiral effective theory. This is one of the main results of this paper.

\subsection{Discretization effects}

Since we use fermions with an exact chiral symmetry, the leading discretization effects in our observables are of order $a^{2}$. We can estimate their magnitude by comparing renormalized dimensionless quantities calculated on the lattices c1 and c3. In Fig. 7 we show the quantity $m r_{0}^{4}\left(\chi_{\nu_{1}}-\chi_{\nu_{2}}\right)$ as a function of $m V /\left(\hat{Z}_{S} r_{0}^{3}\right)$. Within our statistical errors we do not observe significant deviations between the points of the two data sets. A similar behaviour is observed in the other topological sectors. Discretization 

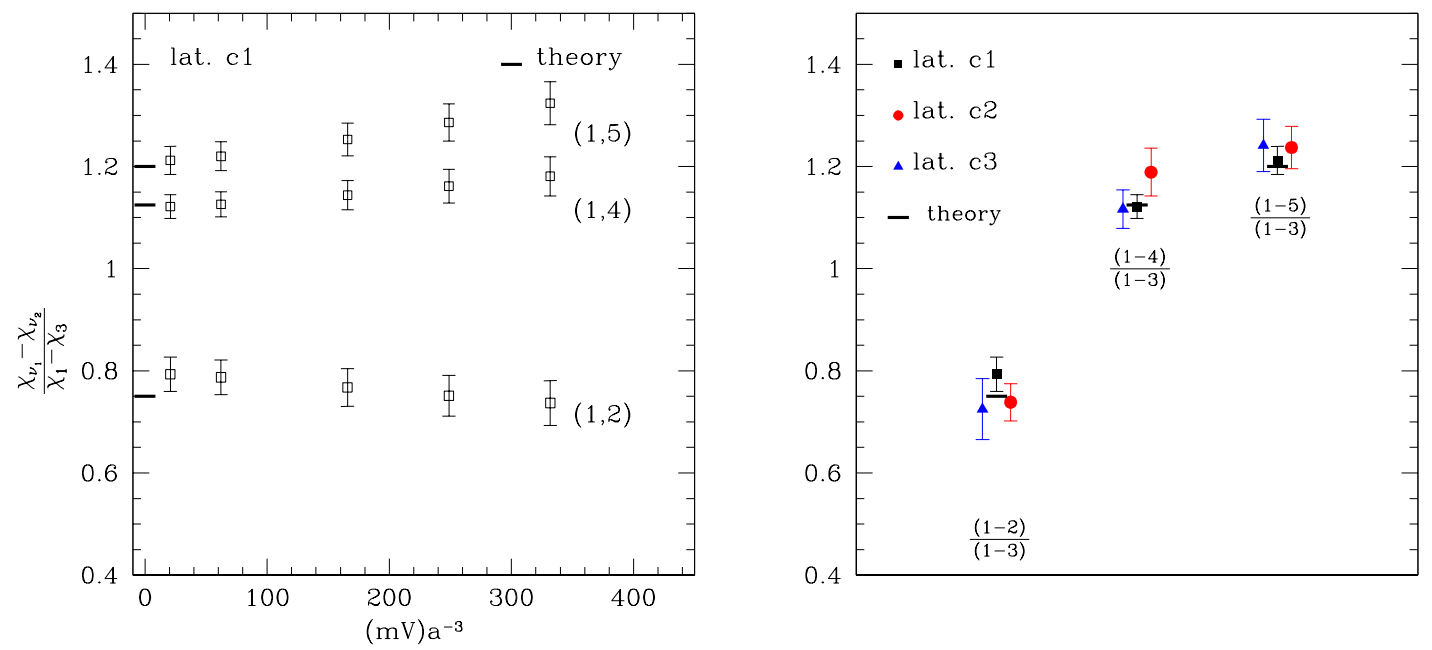

Figure 6: On the left, the ratios $\left(\chi_{\nu_{1}}-\chi_{\nu_{2}}\right) /\left(\chi_{1}-\chi_{3}\right)$ as a function of the quark mass for the lattice $\mathrm{c} 1$ and for $\left(\nu_{1}, \nu_{2}\right)=(1,2),(1,4),(1,5)$. The points at $m=0$ represent the theoretical expectations from the Leutwyler-Smilga sum rule in Eq. (3.6). On the right the same ratios, at the lightest mass available for lattices c1, c2, c3, is compared with the theoretical expectations.

effects are small and are likely to be comparable or below our statistical errors. This is not surprising: quenched computations of various physical quantities carried out with Neuberger fermions show small discretization effects at the lattice spacings of our simulations $[13,54]$. A continuum limit extrapolation of our results is beyond the scope of this paper, and we find it of limited interest before removing the quenched approximation.

\subsection{Extraction of the low-energy constant}

For every lattice and for $\left(\nu_{1}, \nu_{2}\right)=(0,1),(1,2),(2,3)$, we extract the value of the low-energy constant $\Sigma_{\text {eff }} / Z_{S}$ from a one-parameter fit of the observables $\chi_{\nu_{1}}-\chi_{\nu_{2}}$, with the infrared tail truncated as described in Sec. 4, to the corresponding functional form predicted by the chiral effective theory. Combinations with $\nu_{1}, \nu_{2} \geq 4$ have larger statistical errors and are not considered in this analysis. The fit always reproduces the data very well, and the uncorrelated $\chi^{2} /$ d.o.f. are typically very small. The results for $\Sigma_{\text {eff }} / Z_{S}$ are reported in Tab. 3. At small values of $(m V)$ the quenched effective theory predicts a logarithmic behaviour of the form $(m V) \log (m V)$ in $\chi_{0}$, which is clearly seen in our data. If this term is removed from the fit function, the latter is not compatible with the data anymore. For illustration in Fig. 8 the function $\tilde{\chi}_{\nu_{1}}-\tilde{\chi}_{\nu_{2}}$ is superimposed on the data for $\chi_{\nu_{1}}-\chi_{\nu_{2}}$ from lattice $\mathrm{c} 1$.

The values of $a^{3} \Sigma_{\text {eff }} / Z_{S}$ are in good agreement within each data set, a consequence 


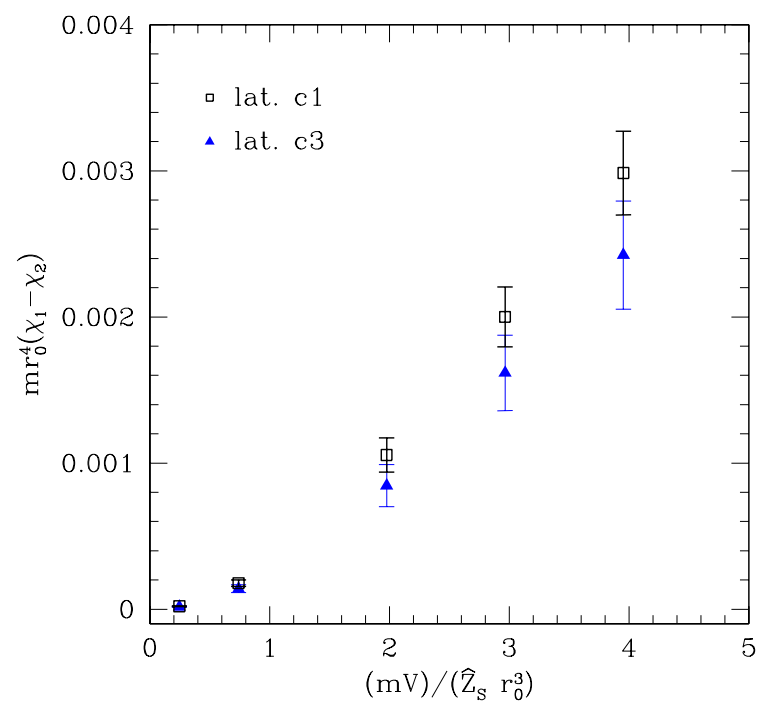

Figure 7: The quantity $m r_{0}^{4}\left(\chi_{\nu_{1}}-\chi_{\nu_{2}}\right)$ as a function of $m V /\left(\hat{Z}_{S} r_{0}^{3}\right)$ for the lattices c1 and $\mathrm{c} 3$.

\begin{tabular}{|ccc|}
\hline lat & $\nu_{1}-\nu_{2}$ & $a^{3} \Sigma_{\text {eff }} / Z_{S}$ \\
\hline c1 & $0-1$ & $0.0040(6)$ \\
& $1-2$ & $0.0039(3)$ \\
& $2-3$ & $0.0034(3)$ \\
\hline c2 & $0-1$ & $0.0035(8)$ \\
& $1-2$ & $0.0049(9)$ \\
& $2-3$ & $0.0040(5)$ \\
\hline c3 & $0-1$ & $0.0015(3)$ \\
& $1-2$ & $0.00178(18)$ \\
& $2-3$ & $0.00188(12)$ \\
\hline
\end{tabular}

Table 3: Results for $a^{3} \Sigma_{\text {eff }} / Z_{S}$ as determined from a fit of the lattice data (see text).

of the fact that the effective theory reproduces the topology dependence observed in the data. The results from lattices $\mathrm{c} 1$ and $\mathrm{c} 2$ confirm that, within our statistical errors, we do not observe a volume dependence in $\Sigma_{\text {eff }}$. By taking $\alpha=0$, the topological susceptibility from Ref. [57] and the quenched value of $F$ from Ref. [29], the NLO formula in Eq. (3.11) suggests for $\Sigma_{\text {eff }}(L \simeq 2.0 \mathrm{fm}) / \Sigma_{\text {eff }}(L \simeq 1.5 \mathrm{fm})$ a positive deviations from 1 of the order of 10 per cent. This is comparable to our statistical uncertainty, and the same holds if 


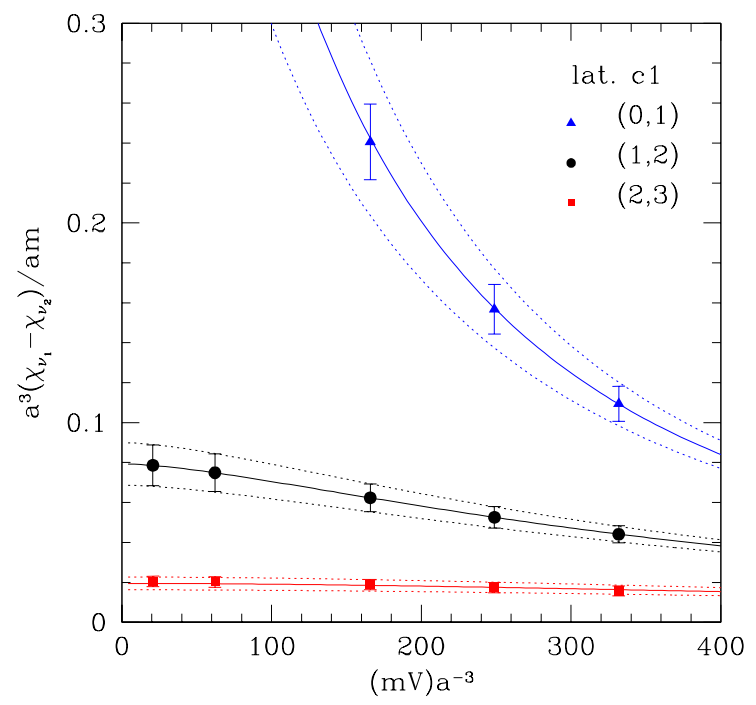

Figure 8: The function $\tilde{\chi}_{\nu_{1}}-\tilde{\chi}_{\nu_{2}}$ is superimposed on data for $\chi_{\nu_{1}}-\chi_{\nu_{2}}$ from lattice c1.

we vary $\alpha$ within a reasonable range of values 4 . Our best result for the renormalization group invariant condensate from the lattices $\mathrm{c} 1$ and $\mathrm{c} 3$ is for the combination $\left(\nu_{1}, \nu_{2}\right)=$ $(1,2)$, while from lattice $\mathrm{c} 2$ is for $(2,3)$ :

$$
\begin{aligned}
& \left(\hat{\Sigma}_{\text {eff }} r_{0}^{3}\right)(L=1.5 \mathrm{fm})=0.33(3) \quad \mathrm{c} 1, \\
& \left(\hat{\Sigma}_{\text {eff }} r_{0}^{3}\right)(L=2.0 \mathrm{fm})=0.34(5) \quad \mathrm{c} 2, \\
& \left(\hat{\Sigma}_{\text {eff }} r_{0}^{3}\right)(L=1.5 \mathrm{fm})=0.29(3) \quad \mathrm{c} 3 \text {. }
\end{aligned}
$$

We take as our best estimate of the condensate at $L=1.5 \mathrm{fm}$ the result from lattice c3, which is the one with the finer lattice spacing. The latter, converted into the more usual $\overline{\mathrm{MS}}$-scheme at $2 \mathrm{GeV}[58]$, is

$$
r_{0}^{3} \Sigma_{\mathrm{eff}}^{\overline{\mathrm{MS}}}(2 \mathrm{GeV})=0.40(4) \text { at } \mathrm{L}=1.5 \mathrm{fm},
$$

and if we use the phenomenological value $r_{0}=0.5 \mathrm{fm}$ we obtain

$$
\Sigma_{\mathrm{eff}}^{\overline{\mathrm{MS}}}(2 \mathrm{GeV})=(290 \pm 11 \mathrm{MeV})^{3} \text { at } \mathrm{L}=1.5 \mathrm{fm} .
$$

The errors in Eqs. (5.5) and (5.6) do not include uncertainties due to discretization effects. Our result is in the range expected from previous computations in the quenched approximation of QCD [5-14]. The determinations from the infinite volume regime [5,6, $8,9]$ are affected by NLO chiral corrections which usually are not taken into account, and which are different from those in the finite volume regime. Even tough the comparison

\footnotetext{
${ }^{4}$ Explicitly, one finds $\Sigma_{\text {eff }}(L=2.0 \mathrm{fm}) / \Sigma_{\text {eff }}(L=1.5 \mathrm{fm}) \sim 1+0.09+0.03 \alpha$.
} 
cannot be very accurate, it is reassuring the good agreement of our result with some of these determinations. First exploratory studies of the chiral condensate in the finite volume regime were performed with small volumes and low statistics $[7,10,11]$. This may be the reason why the large statistical fluctuations generated by the very small eigenvalues of the Dirac operator, which were first reported in Ref. [11], did not occur in some of these computations. A yet different method to extract $\Sigma_{\text {eff }}$ was pursued in Ref. [12-14]. They compared the average value of the individual low-lying eigenvalues of the Dirac operator with the prediction of random matrix theory. Even if it relies on the non-trivial assumptions that the single eigenvalue of the Dirac operator renormalizes with $1 / Z_{S}$ and that the random matrix theory reproduces the QCD spectrum in the infrared, the values of $\Sigma_{\text {eff }}$ obtained in these computations are in good agreement with our result.

\section{Conclusions}

The functional form of the (quenched) QCD chiral condensate in the volume range 5-16 $\mathrm{fm}^{4}$ and for masses $\hat{m} \Sigma V \leq 1$ turns out to be in good agreement, within our statistical errors, with the prediction of the chiral effective theory: the volume, mass and topology dependence are well reproduced by our data. Close to the chiral limit we observe a detailed agreement with the first Leutwyler-Smilga sum rule. The low-energy constant $\Sigma_{\text {eff }}$ is then extracted from a matching of the lattice results with the chiral formulas.

A technical progress which made the computation possible is the introduction of a numerical estimator which is stable in the finite volume regime of (quenched) QCD. Very small eigenvalues of the Dirac operator, which do occur in the Monte Carlo history, do not generate large fluctuations, and a finite size scaling study of the condensate becomes feasible all the way down to the chiral limit. We have not tried to exploit the great freedom in the possible choices of the QCD observable, discussed in Sec. 2, which are unambiguously defined in the continuum limit and could lead to a more precise determination of the low-energy constant $\Sigma_{\text {eff }}$. This is beyond the scope of this paper and we leave it to future studies.

This technique is directly applicable to the computation of the condensate in full QCD simulations.

\section{Acknowledgments}

The present paper is part of an ongoing project whose final goal is to extract lowenergy parameters of (quenched) QCD from numerical simulations with GW fermions. We would like to thank P. Hernández, M. Lüscher, C. Pena, P. Weisz, J. Wennekers and H. Wittig for many interesting discussions and for allowing us to use part of the code developed together. Many thanks to M. Lüscher and M. Testa for interesting discussions on the subject of this paper. Our calculations were performed on PC clusters at CILEA, 
at the University of Rome "La Sapienza" and at the University of Valencia. We thankfully acknowledge the computer resources and technical support provided by all these institutions and the University of Milano-Bicocca for its support. S. N. is supported by the Marie Curie contract MEIF-CT-2006-025673 and was partially supported by EC Sixth Framework Program under the contract MRTN-CT-2006-035482 (FLAVIAnet).

\section{A Definitions and conventions}

We consider the Euclidean space-time discretized on a lattice with spacing $a$. Our conventions for the Dirac matrices are

$$
\gamma_{\mu}^{\dagger}=\gamma_{\mu} \quad\left\{\gamma_{\mu}, \gamma_{\nu}\right\}=2 \delta_{\mu \nu}
$$

with

$$
\gamma_{5}=\gamma_{0} \gamma_{1} \gamma_{2} \gamma_{3}=\left(\begin{array}{cc}
1 & 0 \\
0 & -1
\end{array}\right) .
$$

and the chiral projectors defined as $P_{ \pm}=\left(1 \pm \gamma_{5}\right) / 2$. The massless Neuberger-Dirac operator is defined as

$$
D=\frac{1}{\bar{a}}\left\{1+\gamma_{5} \operatorname{sign}(\mathcal{Q})\right\}
$$

where

$$
\mathcal{Q}=\gamma_{5}\left(a D_{W}-1-s\right), \quad|s|<1, \quad \bar{a}=\frac{a}{1+s},
$$

and $D_{W}$ is the Wilson Dirac operator

$$
D_{W}=\frac{1}{2}\left\{\gamma_{\mu}\left(\nabla_{\mu}^{*}+\nabla_{\mu}\right)-a \nabla_{\mu}^{*} \nabla_{\mu}\right\}
$$

The covariant forward and backward derivatives $\nabla_{\mu}$ and $\nabla_{\mu}^{*}$ are

$$
\begin{aligned}
\nabla_{\mu} \psi(x) & =\frac{1}{a}\{U(x, \mu) \psi(x+a \hat{\mu})-\psi(x)\}, \\
\nabla_{\mu}^{*} \psi(x) & =\frac{1}{a}\left\{\psi(x)-U(x-a \hat{\mu}, \mu)^{-1} \psi(x-a \hat{\mu})\right\},
\end{aligned}
$$

where $U(x, \mu) \in \mathrm{SU}(3)$ are the gauge variables and $\hat{\mu}$ is the versor in direction $\mu$. The massive quark propagator is

$$
S_{m}(x, y)=\tilde{\psi}(x) \bar{\psi}(y)=\frac{1}{1-\frac{\bar{a} m}{2}}\left\{D_{m}^{-1}-\frac{\bar{a}}{2}\right\}(x, y),
$$

where

$$
\tilde{\psi}=\left(1-\frac{\bar{a}}{2} D\right) \psi
$$




\section{B Renormalization of the scalar density}

The bare singlet scalar density

$$
S^{0}(x)=\bar{\psi}(x) \tilde{\psi}(x)
$$

needs to be renormalized to make finite its correlation functions with other gauge invariant operators inserted at a physical distance. $S^{0}$ itself and the identity multiplied by proper combinations of the mass matrix are the only operators with dimension $d \leq 3$ and the same transformations properties under the $S U\left(N_{f}\right)_{\mathrm{L}} \otimes S U\left(N_{f}\right)_{\mathrm{R}}$ group. For quark masses degenerate and real, the renormalized operator can then be written as

$$
\hat{S}^{0}(x)=Z_{S}\left[S^{0}(x)+c_{1} m+c_{2} m^{3}\right],
$$

where $Z_{S}$ is the logarithmic-divergent renormalization constant of the scalar density, and $c_{1}$ and $c_{2}$ are subtraction coefficients which at asymptotically large $a$ diverge as $1 / a^{2}$ and $\ln (a)$ respectively 5 . The coefficients $c_{1}$ and $c_{2}$ can be fixed, for example, by requiring that in the infinite volume limit the condensate satisfies

$$
\begin{aligned}
\left.\frac{d}{d \hat{m}}\left\langle\hat{S}^{0}\right\rangle\right|_{\hat{m}=0} & =0, \\
\left.\frac{d^{3}}{d \hat{m}^{3}}\left\langle\hat{S}^{0}\right\rangle\right|_{\hat{m}=0} & =0 .
\end{aligned}
$$

The value of the renormalized condensate for $\hat{m}>0$ is therefore prescription dependent, a well known fact in QCD. The cumulants of the topological charge $Q$ are ultraviolet finite, and thus the distribution of the topological charge as defined in Eq. (2.4) [1820]. This implies that correlation functions of renormalized local operators inserted at a physical distance are finite also in the theory at fixed topology. In particular the combinations

$$
\left\langle\hat{S}^{0}\right\rangle_{\nu_{1}}-\left\langle\hat{S}^{0}\right\rangle_{\nu_{2}}
$$

are unambiguously defined outside the chiral limit, i.e. they are independent on the particular prescription chosen to renormalize the chiral condensate at finite mass.

\section{References}

[1] S. Weinberg, Physica A96 (1979) 327.

[2] J. Gasser and H. Leutwyler, Ann. Phys. 158 (1984) 142.

[3] L. Del Debbio et al., QCD with light Wilson quarks on fine lattices I: First experiences and physics results, hep-lat/0610059.

\footnotetext{
${ }^{5}$ Notice that for $N_{f}=3$ the chiral group allows for a term proportional to $\mathrm{m}^{2}$. Since the topological charge distribution is ultraviolet finite, this term is not ultraviolet divergent.
} 
[4] ETMC Coll., P. Boucaud et al., Dynamical Twisted Mass Fermions with Light Quarks, hep-lat/0701012.

[5] L. Giusti et al., Nucl. Phys. B538 (1999) 249, hep-lat/9807014.

[6] T. Blum et al., Phys. Rev. D69 (2004) 074502, hep-lat/0007038.

[7] P. Hernández, K. Jansen and L. Lellouch, Phys. Lett. B469 (1999) 198, heplat/9907022.

[8] L. Giusti, C. Hoelbling and C. Rebbi, Phys. Rev. D64 (2001) 114508, heplat/0108007.

[9] P. Hernández et al., Nucl. Phys. Proc. Suppl. 106 (2002) 766, hep-lat/0110199.

[10] MILC Coll., T.A. DeGrand, Phys. Rev. D64 (2001) 117501, hep-lat/0107014.

[11] P. Hasenfratz et al., Nucl. Phys. B643 (2002) 280, hep-lat/0205010.

[12] L. Giusti et al., JHEP 11 (2003) 023, hep-lat/0309189.

[13] J. Wennekers and H. Wittig, JHEP 09 (2005) 059, hep-lat/0507026.

[14] W. Bietenholz and S. Shcheredin, Nucl. Phys. B754 (2006) 17, hep-lat/0605013.

[15] G. Colangelo, J. Gasser and H. Leutwyler, Nucl. Phys. B603 (2001) 125, hep$\mathrm{ph} / 0103088$.

[16] J. Zinn-Justin, Quantum field theory and critical phenomena, Int. Ser. Monogr. Phys. 113 (2002) 1.

[17] H. Leutwyler and A. Smilga, Phys. Rev. D46 (1992) 5607.

[18] L. Giusti et al., Nucl. Phys. B628 (2002) 234, hep-lat/0108009.

[19] L. Giusti, G.C. Rossi and M. Testa, Phys. Lett. B587 (2004) 157, hep-lat/0402027.

[20] M. Lüscher, Phys. Lett. B593 (2004) 296, hep-th/0404034.

[21] J.C. Osborn, D. Toublan and J.J.M. Verbaarschot, Nucl. Phys. B540 (1999) 317, hep-th/9806110.

[22] P.H. Damgaard et al., Nucl. Phys. B547 (1999) 305, hep-th/9811212.

[23] M. Lüscher, Comput. Phys. Commun. 165 (2005) 199, hep-lat/0409106.

[24] C. Urbach et al., Comput. Phys. Commun. 174 (2006) 87, hep-lat/0506011.

[25] A. Hasenfratz, P. Hasenfratz and F. Niedermayer, Phys. Rev. D72 (2005) 114508, hep-lat/0506024. 
[26] JLQCD Coll., S. Hashimoto et al., Dynamical overlap fermion at fixed topology, hep-lat/0610011.

[27] C.B. Lang, P. Majumdar and W. Ortner, hep-lat/0611010.

[28] JLQCD Coll., H. Fukaya et al., Two-flavor lattice QCD simulation in the epsilonregime with exact chiral symmetry, hep-lat/0702003.

[29] L. Giusti et al., JHEP 04 (2004) 013, hep-lat/0402002.

[30] L. Del Debbio et al., JHEP 02 (2006) 011, hep-lat/0512021.

[31] H. Neuberger, Phys. Lett. B417 (1998) 141, hep-lat/9707022.

[32] P.H. Ginsparg and K.G. Wilson, Phys. Rev. D25 (1982) 2649.

[33] M. Lüscher, Phys. Lett. B428 (1998) 342, hep-lat/9802011.

[34] K. Fujikawa, Phys. Rev. Lett. 42 (1979) 1195.

[35] K. Fujikawa, Phys. Rev. D21 (1980) 2848, Erratum-ibid. D22 (1980) 1499. .

[36] H. Neuberger, Phys. Rev. D57 (1998) 5417, hep-lat/9710089.

[37] P. Hasenfratz, V. Laliena and F. Niedermayer, Phys. Lett. B427 (1998) 125, heplat/9801021.

[38] T. Banks and A. Casher, Nucl. Phys. B169 (1980) 103.

[39] J. Gasser and H. Leutwyler, Phys. Lett. B184 (1987) 83.

[40] J. Gasser and H. Leutwyler, Phys. Lett. B188 (1987) 477.

[41] R.C. Brower, P. Rossi and C.I. Tan, Phys. Rev. D23 (1981) 942.

[42] C.W. Bernard and M.F.L. Golterman, Phys. Rev. D46 (1992) 853, hep-lat/9204007.

[43] S.R. Sharpe, Phys. Rev. D46 (1992) 3146, hep-lat/9205020.

[44] P.H. Damgaard et al., Nucl. Phys. B629 (2002) 445, hep-lat/0112016.

[45] P.H. Damgaard, Nucl. Phys. B608 (2001) 162, hep-lat/0105010.

[46] P. Hasenfratz and H. Leutwyler, Nucl. Phys. B343 (1990) 241.

[47] L. Giusti et al., Comput. Phys. Commun. 153 (2003) 31, hep-lat/0212012.

[48] L. Giusti et al., JHEP 11 (2004) 016, hep-lat/0407007.

[49] H. Fukaya, S. Hashimoto and K. Ogawa, Prog. Theor. Phys. 114 (2005) 451, heplat/0504018. 
[50] K. Ogawa and S. Hashimoto, Prog. Theor. Phys. 114 (2005) 609, hep-lat/0505017.

[51] L. Giusti and S. Necco, PoS LAT2005 (2006) 132, hep-lat/0510011.

[52] L. Giusti et al., On $K \rightarrow \pi \pi$ amplitudes with a light charm quark, hep-ph/0607220.

[53] P. Hernández, K. Jansen and M. Lüscher, Nucl. Phys. B552 (1999) 363, heplat/9808010.

[54] R. Babich et al., JHEP 01 (2006) 086, hep-lat/0509027.

[55] R. Sommer, Nucl. Phys. B411 (1994) 839, hep-lat/9310022.

[56] ALPHA Coll., M. Guagnelli, R. Sommer and H. Wittig, Nucl. Phys. B535 (1998) 389, hep-lat/9806005.

[57] L. Del Debbio, L. Giusti and C. Pica, Phys. Rev. Lett. 94 (2005) 032003, hepth/0407052.

[58] ALPHA Coll., J. Garden et al., Nucl. Phys. B571 (2000) 237, hep-lat/9906013. 